\title{
Fast curing versus conventional resins - degradation due to hygrothermal and UV exposure
}

\author{
N. M. Barkoula ${ }^{1 *}$, M. Karabela ${ }^{2}$, N. E. Zafeiropoulos ${ }^{2}$, P. Tsotra ${ }^{3}$ \\ ${ }^{1}$ University of Ioannina, Department of Materials Science and Engineering, Composite and Smart Materials Laboratory, \\ 45110 Ioannina, Greece \\ ${ }^{2}$ University of Ioannina, Department of Materials Science and Engineering, Polymer's Laboratory, 45110 Ioannina, \\ Greece \\ ${ }^{3}$ University of Applied Sciences and Arts Northwestern Switzerland FHNW, School of Engineering, Institute of Polymer \\ Engineering, 5210 Windisch, Switzerland
}

Received 9 September 2019; accepted in revised form 15 November 2019

\begin{abstract}
In the current paper, three resin systems are investigated, the first is a high temperature fast curing system, the second is a high-temperature system that requires several hours to cure and the third is a slow room temperature curing system. All systems are exposed to hygrothermal and combined moisture/temperature/UV protocols, and their durability is assessed using Fourier transform infrared (FTIR) spectroscopy, mechanical and thermomechanical characterization. The conventional slow curing system presents a wide distribution of polymer chains that leads in excess free volume/increased susceptibility to water. The medium curing system is more homogenous and shows lower absorption profiles. The fast curing system shows two network domains, one that is more crosslinked and contributes towards higher free volume/water absorption and the other that is partially cured and supports chain scission/leaching processes during exposure. Continuation of crosslinking prevails in conventional resins upon hygrothermal and combined UV exposure. At the same time antagonistic effects (i.e. continuation of the crosslinking process versus plasticization, chain scission, and leaching) are observed in medium curing and fast curing systems upon exposure.
\end{abstract}

Keywords: thermosetting resins, fast curing, environmental exposure, mean molecular weight between crosslinks, glass transition temperature

\section{Introduction}

Epoxy resins are a class of materials of great significance since they can be used stand-alone for the manufacturing of versatile products, but most importantly they serve as matrix in fiber-reinforced polymer composites that find application in various sectors, such as aerospace, wind energy, marine industry, etc [1]. One of the key parameters to obtain highperformance epoxy systems/composites is related to the curing process during which the liquid components of the resin system are converted into a rigid polymer network with increased stiffness, strength, and temperature/dimensional stability [1,2]. Typically in a two-component system, this occurs by allowing the resin to react with the hardener for several hours depending on the chemistry of the system and the applied temperature. In order to expedite processing, a new class of resin systems, namely fast curing ones, has been introduced by the chemical industry [3-11]. The target of the resin industry is to design fast curing systems that possess low viscosity, long pot life, and satisfactory performance. Among various types of accelerators, amine-based hardeners (aliphatic, alicyclic and aromatic) seem to simultaneously support 
fast curing, easy molding, and advanced mechanical performance [9-11]. Such systems are of great importance since they can be manufactured using various techniques, i.e. injection molding, infusion, resin transfer molding, resulting in finished parts with reduced processing time. Contrary to conventional ones, the chemistry of fast curing resin systems allows their conversion to a crosslinked system within few minutes by the application of relatively high temperatures. However this conversion is highly exothermic, and due to the low thermal conductivity of the epoxy systems it may influence the curing process [8], resulting in inhomogeneity, residual stresses, and microvoids.

Another key aspect of the applicability of resin systems is their environmental durability [12-20]. It is well accepted that epoxy resins show reversible and irreversible changes to their physical and chemical properties after exposure to environmental conditions such as moisture, increased temperature and/or $\mathrm{UV}$ radiation. The response to those environments is not only related to their chemical nature but also their cure state. As widely documented, hydro/hygrothermal exposure of epoxy resins may result in plasticization (reversible) and chemical reactions, such as hydrolysis, leaching of partially cured molecules, and post-curing (irreversible) [12-18]. At the same time, UV radiation may have adverse effects on the properties of epoxy systems since it can result in the continuation of the crosslinking process in partially cured systems, while prolonged exposure is associated with chain scission, micro-cracking, and brittleness $[12,19,20]$.

Since the curing process is central for the performance of epoxy-based systems, the current study is the first to compare the degradation behavior of conventional and fast curing epoxy resin systems after hygrothermal exposure and combined UV radiation. The first system tested here is a conventional room temperature slow curing one; the second system is a warm system that requires several hours to cure fully, while the third system is a fast curing one that crosslinks within few minutes at high temperatures. In order to verify their environmental durability all systems were formulated according to the manufacturer's recommendations and exposed to hygrothermal loading at three different temperatures. Another set of specimens was degraded via combined UV, temperature and moisture exposure. The effect of environmental exposure on the durability of the prepared systems was assessed by means of water absorption, FTIR, mechanical and thermomechanical characterization.

\section{Experimental}

\subsection{Materials - curing protocol - specimen's preparation}

Three different two-part epoxy systems were used in the current study. The designation, resin, hardener, stoichiometry and curing protocol followed for the preparation of each system are presented in Table 1. All systems were kindly supplied by Huntsman Advanced Materials, Basel, Switzerland. The first tested system belongs to the cold-curing ones and comprises of Araldite ${ }^{\circledR}$ LY5052 epoxy resin with an Aradur $^{\circledR} 5052$ hardener. Due to the slow curing protocol used in this system, it is designated as slow curing (SC). The second is a warm curing epoxy system that consists of Araldite ${ }^{\circledR}$ LY 1564 and Aradur ${ }^{\circledR} 3486$ hardener. This system has a medium curing duration at higher temperatures and is designated as medium curing (MC). The last one is a warm fast curing (FC) epoxy system that consists of Araldite ${ }^{\circledR}$ LY 3585 and XB 3458 hardener. According to the manufacturer the chemical basis of Araldite ${ }^{\circledR}$ LY5052 is phenol polymer with formaldehyde, glycidylether and 1,4Bis(2,3-epoxypropoxy)butane, while Araldite ${ }^{\circledR} \mathrm{LY}$ 1564 and Araldite ${ }^{\circledR}$ LY 3585 are mainly composted of Bisphenol A. Aradur ${ }^{\mathbb{R}} 5052$, $\operatorname{Aradur}^{\mathbb{R}} 3486$ and XB 3458 are amine-based hardeners.

For the preparation of specimens specific quantities (based on the stoichiometry of the manufacturer, see Table 1) of the resin/hardener were accurately weighted using a Sartorius $( \pm 0.001 \mathrm{mg})$ balance (Sartorius Lab Instruments GmbH, Goettingen, Germany) and placed at $R T$ for $30 \mathrm{~min}$ in a vacuum oven (Vacutherm, Thermo Fisher Scientific, Waltham,

Table 1. Designation, resin/hardener type and stoichiometry, as well as curing protocol used for the preparation of epoxy systems.

\begin{tabular}{|c|l|c|c|l|}
\hline Designation & \multicolumn{1}{|c|}{ Resin } & Hardener & Resin : Hardener weight ratio & \multicolumn{1}{|c|}{ Curing protocol } \\
\hline SC & Araldite $^{\circledR}$ LY 5052 & Aradur $^{\circledR} 5052$ & $100: 38$ & 2 days at $25^{\circ} \mathrm{C}$ \\
\hline MC & Araldite $^{\circledR}$ LY 564 & Aradur $^{\circledR} 3486$ & $100: 34$ & 8 hours at $80^{\circ} \mathrm{C}$ \\
\hline FC & Araldite $^{\circledR}$ LY 3585 & XB 3458 CH & $100: 19$ & 3 min at $100^{\circ} \mathrm{C}$ \\
\hline
\end{tabular}


Massachusetts, United States) in order to remove any moisture/bubbles. The resin was then hand mixed with the hardener and placed to an open silicon rubber mold. The mold was then transferred in the aforementioned vacuum oven, and the epoxy systems were cured according to the protocol presented in Table 1.

\subsection{Environmental exposure}

Two different exposure protocols were used in the current study. In the case of water exposure, five specimens per tested system were fully immersed in a water bath (Grant Instruments, Cambridge, United Kingdom) filled with distilled water for up to $2160 \mathrm{~h}$ following the procedure described in ASTM D570 98 [21]. Since the mobility of the water and the relaxation phenomena in the polymer network are temperature dependent, water absorption studies have been conducted at both room and elevated temperatures according to previous publications [22-26]. More specifically, in the current study specimens were exposed at three different temperatures, i.e. 23, 50 and $70^{\circ} \mathrm{C}[22,25]$. Prior to the exposure the specimens were dried at $50^{\circ} \mathrm{C}$ until they reached a constant initial weight $\left(W_{0}\right)$ [21]. During the exposure, the specimens were removed from the water bath at specific time intervals, wiped and weighted with the aforementioned precision balance $\left(W_{\mathrm{i}}\right)$. The $\%$ weight gain of the specimens $\left(\Delta W / W_{0}\right)$ was calculated using Equation (1):

$\frac{\Delta W}{W_{0}}=\frac{W_{\mathrm{i}}-W_{0}}{W_{0}}$

In the case of the combined moisture - temperature - UV exposure five specimens per tested system were placed in a house-made environmental chamber with UV lamps (UV A - 13.6 W, UV B - 3 W), temperature and moisture control and exposed to the following cycle: $8 \mathrm{~h} \mathrm{UV}$ at $60^{\circ} \mathrm{C}$ кal $4 \mathrm{~h}$ condensation at $50^{\circ} \mathrm{C}$, following the procedure described in ASTM D4329 [27]. A set of specimens was removed after $1000 \mathrm{~h}$ of exposure while another was exposed for $2000 \mathrm{~h}$ in total. Horizontal and vertical reposition was followed every week in order to minimize any effects from temperature or UV light variation [27].

\subsection{Characterization}

\subsubsection{FTIR}

FTIR-ATR spectra were recorded using an iD7 ATR spectrometer (Thermo Fisher Scientific, Waltham,
Massachusetts, United States) in the range of 4000$400 \mathrm{~cm}^{-1}$. For each sample, 32 scans were obtained at a resolution of $4 \mathrm{~cm}^{-1}$.

\subsubsection{Mechanical testing}

Dogbone specimens with gauge dimensions of $42 \times 4 \times 2 \mathrm{~mm}^{3}$ were used for the evaluation of the tensile behavior of the prepared samples before and after the combined UV exposure. Three to five samples were tensioned at a crosshead speed of $10 \mathrm{~mm} / \mathrm{min}$ using a mini-tester (Ernest F. Fullam, Inc. New York, US) with a $1000 \mathrm{~N}$ load cell. Based on the obtained results, statistical calculations (mean values and standard deviation) were performed.

\subsubsection{Dynamic mechanical analysis}

Dynamic mechanical analysis (DMA) studies were performed on rectangular $60 \times 10 \times 4 \mathrm{~mm}^{3}$ specimens using a Q800 analyzer (TA Instruments, New Castle, US) under dual cantilever configuration. The specimens were exposed to a temperature scan from 30 to $180^{\circ} \mathrm{C}$ at a rate of $2^{\circ} \mathrm{C} / \mathrm{min}$. The deformation amplitude was set at $15 \mu \mathrm{m}$, and the applied frequency was $1 \mathrm{~Hz}$.

\section{Results and discussion}

\subsection{Water exposure}

The water absorption profile of the three epoxy systems is presented in Figure 1 as a function of exposure temperature and duration. Overall the three investigated systems show a Fickian behavior [28], i.e. a linear increase of water absorption with $t^{1 / 2}$ during the early stage of absorption, followed by decreased rate of absorption at intermediate times and finally saturation after prolonged exposure. It is interesting to note that the second and third stages are only observed at higher water temperatures. More specifically, as observed in Figure 1a, the amount of absorbed water increases linearly with $t^{1 / 2}$ when the water temperature is $23^{\circ} \mathrm{C}$ (Figure 1a) while specimens do not reach saturation within the duration of the experiment $(2160 \mathrm{~h}=3$ months). On the other hand immersion in water with higher temperatures $\left(50^{\circ} \mathrm{C}\right.$ - Figure $1 \mathrm{~b}$ and $70^{\circ} \mathrm{C}-$ Figure $\left.1 \mathrm{c}\right)$ results in higher diffusivity and saturation within the time scale of the experiments. It is well accepted that the first linear part is diffusion controlled, while the second part of the curve denotes a competition between diffusion and relaxation phenomena, which dominate during the third (equilibrium) stage [22, 29]. 
Thus it can be concluded that all three systems do not show any significant network relaxation at low temperatures of water exposure. This is because they are in the glassy state and relaxation phenomena are much slower compared to diffusion at $R T$ [29]. Next to that, in all cases the $\mathrm{SC}$ system shows the highest level of water absorption followed by the $\mathrm{MC}$ and FC ones. Another interesting observation is that while the diffusivity of the MC system coincides with that of the SC, the \% water absorption at saturation is similar to that of the FC system (Figure $1 \mathrm{~b}$ and 1c). In order to quantify the differences in the response of the three systems, their water absorption profiles are fitted using the Fick's law [28] according to Equation (2) and the procedure described in detail elsewhere [24]:

$$
\frac{\Delta W}{W_{0}}=\frac{4 W_{\infty}}{\pi^{1 / 2}}\left(\frac{D t}{\alpha^{2}}\right)^{1 / 2}
$$

where $W_{\infty}$ is the \% water absorbed at saturation, $D$ is the diffusion coefficient in $\left[\mathrm{m}^{2} / \mathrm{s}\right], \alpha$ is the thickness of the specimens in [m], and $t$ is the time in [s]. Solving Equation (2) it is possible to calculate the diffusion coefficient $D$ following Equation (3):

$D=\left(\frac{\Delta W}{W_{0}}\right)^{2} \frac{1}{t} \frac{\pi \alpha^{2}}{16 W_{\infty}^{2}}=S^{2} \frac{\pi \alpha^{2}}{16 W_{\infty}^{2}}$

where $S$ is the slope [\%/s] of the linear part of the absorption curve versus square root of time.

Based on Equation (3) and Figure 1, the diffusion coefficient and \% absorbed water after $2160 \mathrm{~h}$ of immersion are calculated and presented in Figure 2a and Figure 2b, respectively. It is important to note that it is not accurate to calculate the diffusion coefficient after water immersion at $23^{\circ} \mathrm{C}$ since none of the exposed systems reached saturation; however, for comparison reasons only a coefficient has been estimated considering the $\%$ absorbed water after $2160 \mathrm{~h}$ as saturation in this particular case. As illustrated in Figure 2a, the diffusion of water is very slow at $23^{\circ} \mathrm{C}$ and increases as the temperature of water rises to 50 and $70^{\circ} \mathrm{C}$. The highest diffusion coefficient is observed at $70^{\circ} \mathrm{C}$ for the $\mathrm{MC}$ system, while for the same exposure conditions the FC system shows the lowest one (app. 2 times higher $D$ in the case of the MC system). At intermediate exposure temperatures $\left(50^{\circ} \mathrm{C}\right)$ the $\mathrm{MC}$ system presents the highest diffusivity while the $\mathrm{SC}$ and the $\mathrm{FC}$ ones behave similarly. Equally to the diffusion coefficient, the total amount of absorbed water after $2160 \mathrm{~h}$ of
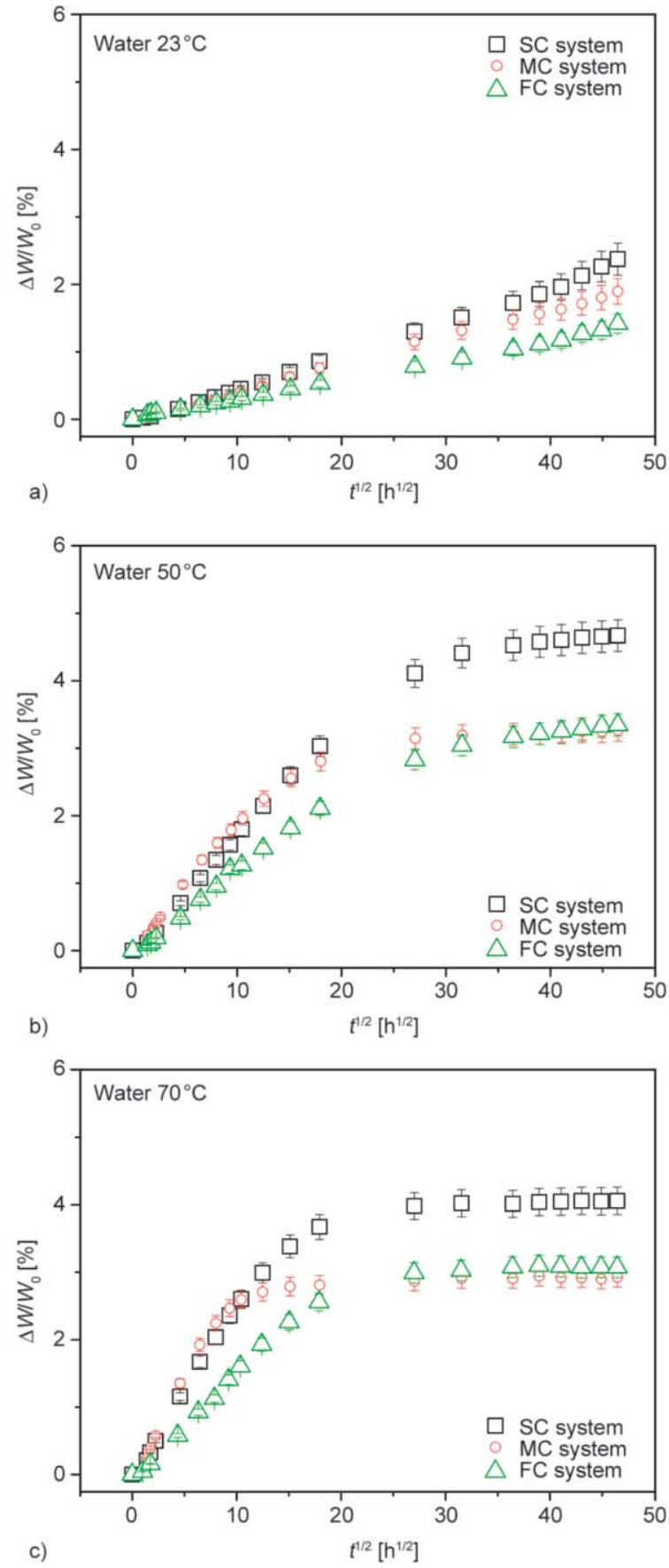

Figure 1. Water absorption of SC, MC and FC systems as a function of time after full immersion at a) $23^{\circ} \mathrm{C}$, b) $50^{\circ} \mathrm{C}$ and c) $70^{\circ} \mathrm{C}$.

exposure (saturation in the case of 50 and $70^{\circ} \mathrm{C}$ ) is lower after immersion in water at $23^{\circ} \mathrm{C}$, and increases as the water temperature rises (Figure $2 b$ ). Interestingly, the total amount of absorbed water at $50^{\circ} \mathrm{C}$ is slightly higher than that at $70^{\circ} \mathrm{C}$. The $\mathrm{SC}$ system contains the highest amount of water (4-4.6\%), while the $\mathrm{MC}$ and the FC ones hold the same level of water at saturation (2.9-3.1\%). As discussed previously the obtained differences in the diffusion coefficient and $\%$ absorbed water of the three tested systems may be 

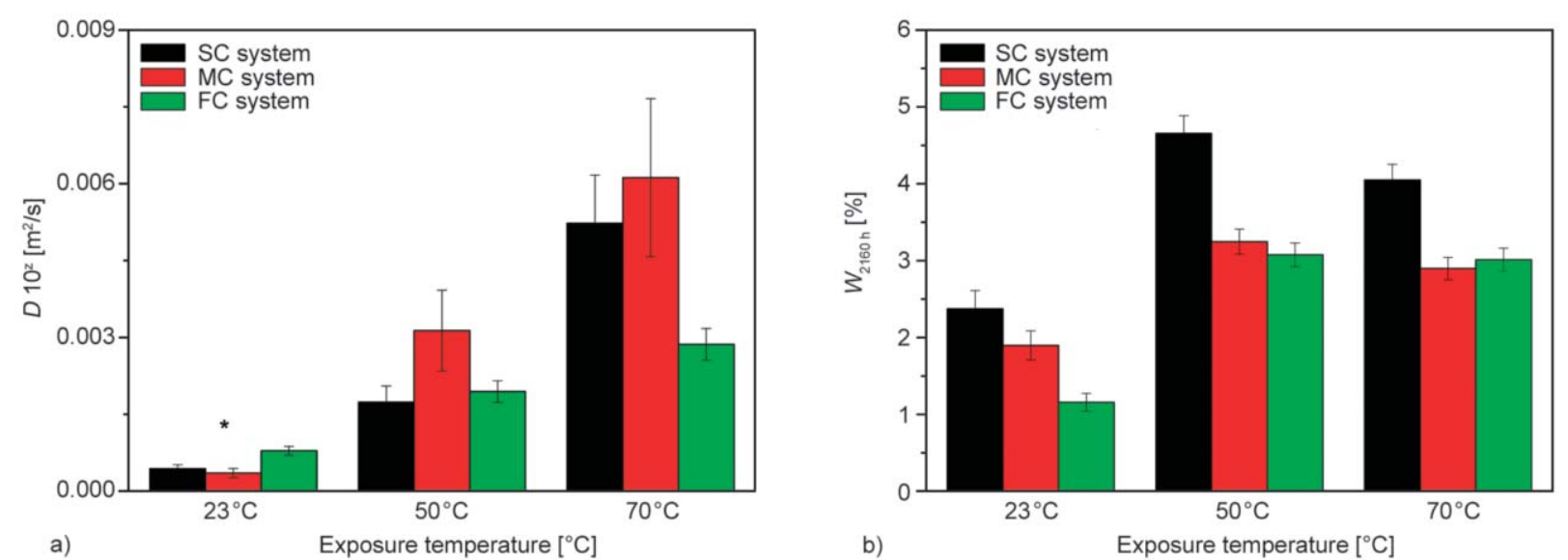

Figure 2. a) Diffusion coefficient $(D)$ and b) $\%$ absorbed water after full immersion $\left(W_{2160 \mathrm{~h}}\right)$ at 23,50 and $70{ }^{\circ} \mathrm{C}$ of $\mathrm{SC}, \mathrm{MC}$ and FC systems.

*Note that the estimation of the diffusion coefficient at $23^{\circ} \mathrm{C}$ is valid only for comparison reasons since this system did not reach saturation within the time scale of the experiment.

associated with their free volume as well as with their affinity to water [22-26, 29]. The free volume is, in turn related to the crosslink density, $T_{\mathrm{g}}$, and degree of cure, while the affinity to water is determined by the ability of the polar groups of the resin to interact with water molecules and form hydrogen bonds [22-26, 29]. It has been reported that an increase in the degree of cure results in higher amounts of absorbed water, and this has been related to a corresponding increase in the free volume upon curing [22]. The amount of free volume also controls the access of water to the polar groups of the epoxy system. It is, however, important to bear in mind that the free volume of cured epoxy changes during hygrothermal aging, and the same holds for the available polar sites of the resin [22]. More information regarding the existence of free and bound water, as well as crosslinking $/ T_{\mathrm{g}}$ could be obtained from FTIR and DMA data. Thus the overall discussion of the obtained behavior follows at the end of this paragraph. FTIR spectra of the SC, MC and FC systems, as a function of the immersion conditions, are shown in Figure 3, respectively. Spectra of samples obtained straight after mixing the resin with the hardener (before curing) and after curing but before exposure are added in Figure 3 for reference. In accordance with previous observations all systems display characteristic vibrational modes of various functional groups in the area of $4000-400 \mathrm{~cm}^{-1}$ wavenumbers [30-37]. The analysis that follows focuses on specific peaks that signify alterations in the crosslinking process and/or denote changes related to degradation due to environmental exposure.
More specifically, it is possible to identify deformation modes of phenyl groups at 830-820, 1250$1170 \mathrm{~cm}^{-1}$, ether stretching at $1036 \mathrm{~cm}^{-1}$, aromatic ring stretching at $1509 \mathrm{~cm}^{-1}$, alkyl units stretching at $2990-2800 \mathrm{~cm}^{-1}$, and a very broad shoulder at app. $3400 \mathrm{~cm}^{-1}$ which can be associated with $-\mathrm{OH}$ and $-\mathrm{NH}$ stretching and existence of intermolecular hydrogen bonds [30, 33-37]. At the same time, the SC and $\mathrm{MC}$ systems present a characteristic peak at $916 \mathrm{~cm}^{-1}$ before curing, which is indicated of unreactive epoxy (Figure 3a and Figure 3b) [33-35, 38]. After curing, this peak transforms into a small shoulder at the same location for all exposure conditions (Figure $3 \mathrm{a}$ and Figure $3 \mathrm{~b}$ ) which suggests that the crosslinking of the system is almost complete before exposure. Interestingly the FC system does not present a strong peak at $916 \mathrm{~cm}^{-1}$ before curing (Figure $3 \mathrm{c}$ ) while a shoulder is apparent after curing/exposure. It can be speculated that due to the fast curing nature of the resin, the curing reaction has proceeded during mixing, hindering the clear observation of the oxirane group of the epoxy resin. Furthermore, in all three systems it can be observed that the aforementioned shoulder varies with the exposure conditions, suggesting a potential influence of the exposure conditions on the crosslinking process, which needs to be verified by the DMA data.

Although the spectra of the tested systems present overall great similarities before and after exposure, an attempt is made here to identify key differences in their water-related bands. Water diffusion in epoxy systems has been widely studied using FTIR [29, 34, 38-42]. It has been stated that stretching of water 

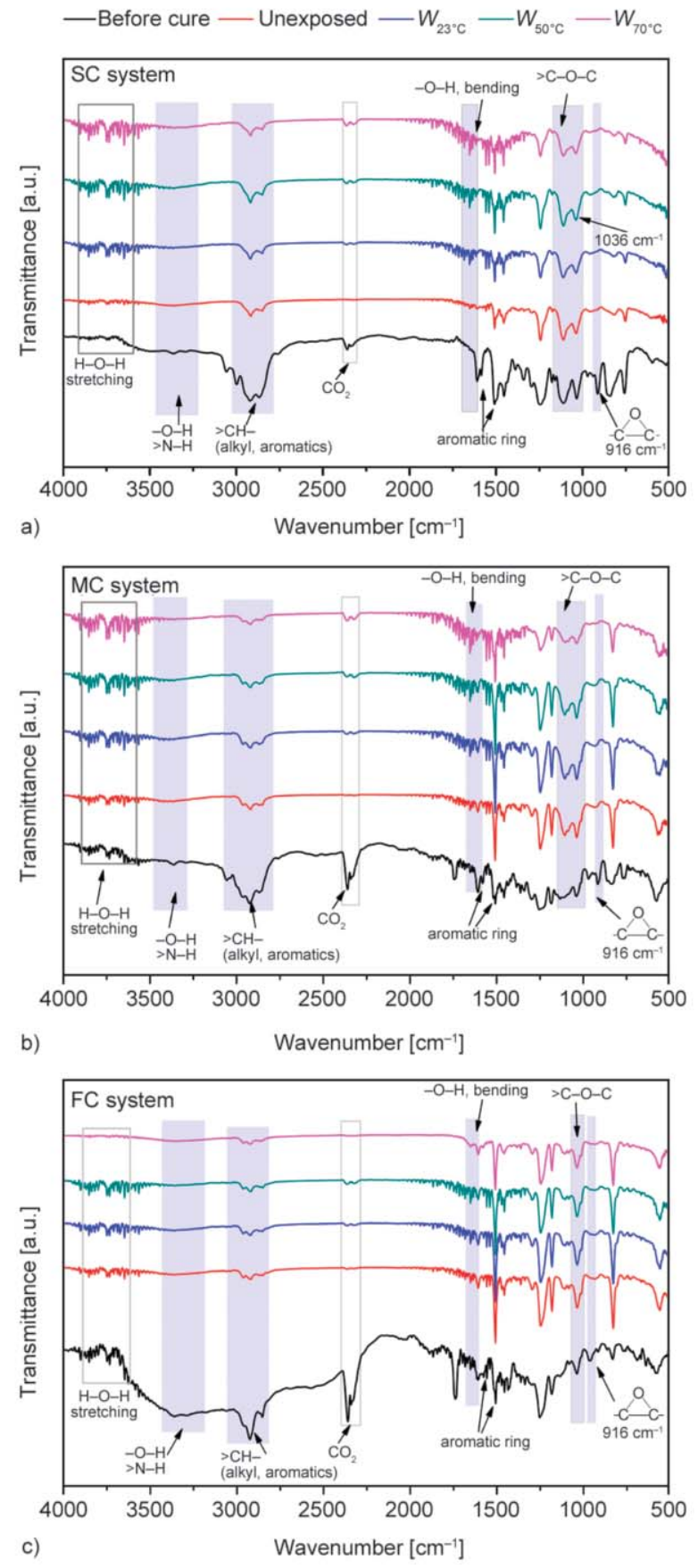

Figure 3. FTIR-ATR spectra of a) SC, b) MC and c) FC systems before and after water exposure at various temperatures; spectra of uncured systems are added for reference.

molecule can be found at $3900-2800 \mathrm{~cm}^{-1}$, while bending is located at app. $1640 \mathrm{~cm}^{-1}[29,34,37-40]$. It should be however noted that other studies connect the band found at $1630 \mathrm{~cm}^{-1}$ with the stretching vibration of $\mathrm{C}=\mathrm{C}$ bond and the absence of the band with completion of the crosslinking process [36]. Furthermore, most researchers agree that water can be found in a free state, which is reflected by a band at app. $3600 \mathrm{~cm}^{-1}$ and a hydrogen-bonded one, which gives a broad band at app. $3400-3300 \mathrm{~cm}^{-1}[29,34$, 38-41]. Figure 3 reveals higher intensity fluctuations in the spectra of the investigated systems $>3600 \mathrm{~cm}^{-1}$ and around $1645 \mathrm{~cm}^{-1}$, especially after water exposure. The obtained signal is very weak around $3300 \mathrm{~cm}^{-1}$, and does not vary significantly with the exposure conditions/tested system. Thus based on the previous analysis it seems that water exists in a free state, and, in line with the water absorption results, its amount increases with water temperature. In terms of changes in the spectra due to degradation, previous studies have focused on the peaks at 1660 and $1558 \mathrm{~cm}^{-1}$, and the one at $1509 \mathrm{~cm}^{-1}$. The former peaks are connected with the vibration of $\mathrm{NH}$ groups and the leaching of degradation products. The latter is attributed to the $-\mathrm{NH}$ deformation of the secondary amine in the crosslinker and is associated with chain scission and alteration in the crosslinking process of the exposed system [38, 42]. Using the intensity of the peak at $830 \mathrm{~cm}^{-1}$ as reference [30], the relative intensity of the aforementioned peaks has been calculated for each system before and after water immersion. The general trend is an increase of the relative intensity of the peaks upon exposure for the SC system, while fluctuations are observed in the values of the MC and FC systems. It should be noted however that the obtained changes are minor and therefore cannot lead to safe conclusions. Finally, the doublet of bands located at 2360 and $2340 \mathrm{~cm}^{-1}$ seen in all spectra correspond to $\mathrm{CO}_{2}$ and does not relate to any epoxy reaction/ degradation products.

To evaluate the influence of water exposure on the viscoelastic response of the investigated systems, the storage modulus $\left(E^{\prime}\right)$ and loss factor $(\tan \delta)$ are plotted in Figure 4 as a function of temperature. All investigated systems present a typical response of thermosetting resins, i.e. a small decrease of the $E^{\prime}$ in the glassy region followed by an abrupt reduction in the glass - rubbery transition zone, which sets of to an almost constant value in the rubbery region. At the same time, as the temperature increases, the damping $(\tan \delta)$ is low in the glassy and rubbery states and passes through a maximum in the transition zone. The maximum of the $\tan \delta$ curve denotes the glass transition temperature $\left(T_{\mathrm{g}}\right)$, while its position and height provide information regarding the structure of the investigated systems [43]. For direct comparison of the three epoxy systems, critical viscoelastic properties, such as the $E^{\prime}$ in the glassy $\left(E^{\prime}{ }_{\mathrm{G}}\right)$ and rubbery state 
$\left(E_{\mathrm{R}}^{\prime}\right)$ and $T_{\mathrm{g}}$ are included in Table 2. The $E_{\mathrm{G}}^{\prime}$ of all investigated systems has been defined at $35^{\circ} \mathrm{C}$ while the $E_{\mathrm{R}}^{\prime}$, at $T_{\mathrm{g}}+50^{\circ} \mathrm{C}$. Since the glass to rubbery transitions in same cases are very broad, the exact location of the peaks of the $\tan \delta$ curves has been obtained using signal processing.

As observed in Figure 4a and Table 2, the unexposed SC system presents $E_{G}^{\prime}$ values in the range of $2500 \mathrm{MPa}$, which are typical for epoxy systems [4, $5,19,20,24,25]$. Water exposure results in up to $16 \%$ deterioration of the $E^{\prime}{ }_{\mathrm{G}}$ while the $E_{\mathrm{R}}^{\prime}$ is slightly
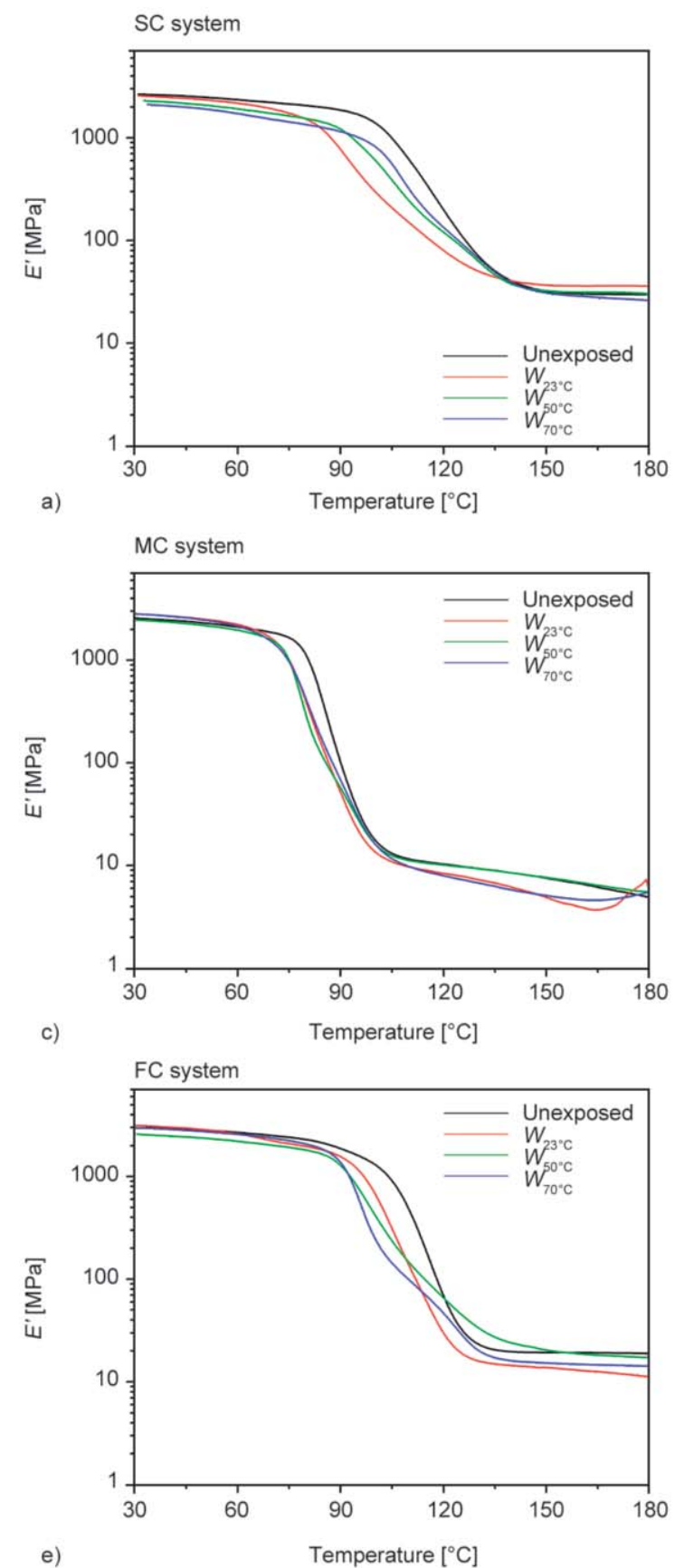

improved, especially after immersion at water of $23{ }^{\circ} \mathrm{C}$ (see Table 2). Slight changes of the $E_{\mathrm{G}}^{\prime}$ are not connected with the mobility of the SC system, since below $T_{\mathrm{g}}$ chains are more or less rigid [44]. On the contrary, increase in the $E_{\mathrm{R}}^{\prime}$ of the SC system could be attributed to higher crosslink density, which restrains the mobility in the rubbery region [44]. At the same time the unexposed SC system shows a very broad glass to rubbery transition, with a peak at $113^{\circ} \mathrm{C}$ as observed in the tan $\delta$ spectra (Figure $4 \mathrm{~b}$ ). Overall, very broad transitions denote very wide
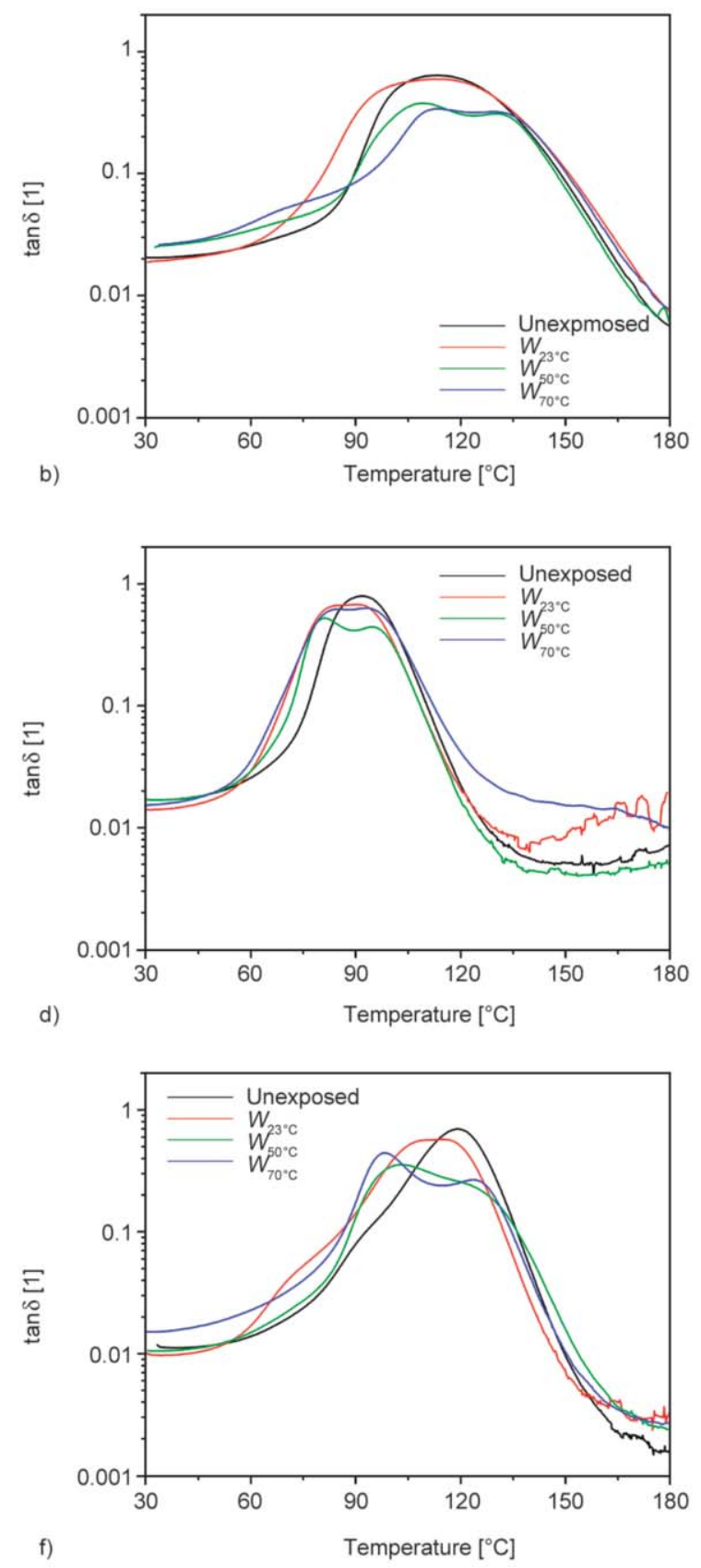

Figure 4. Storage modulus $\left(E^{\prime}\right)$ and loss factor $(\tan \delta)$ of SC: a), b), MC: c), d) and FC: e), f) systems, respectively before and after water exposure at various temperatures. 
Table 2. Glass transition temperature $\left(T_{\mathrm{g}}\right)$, storage modulus in the glassy region $\left(E_{\mathrm{G}}^{\prime}\right)$ and rubbery plateau $\left(E_{\mathrm{R}}^{\prime}\right)$ and mean molecular weight between crosslinks $\left(M_{\mathrm{c}}\right)$ before exposure, after water immersion and after combined UV exposure of SC, MC and FC systems. Note that U stands for unexposed specimens, W23, W50 and W70 for those immersed in water at the corresponding temperatures, while UVI and UVII for combined UV exposure for 1000 and 2000h, respectively.

\begin{tabular}{|c|c|c|c|c|c|c|c|c|c|c|c|c|}
\hline & \multicolumn{4}{|c|}{ SC system } & \multicolumn{4}{|c|}{ MC system } & \multicolumn{4}{|c|}{ FC system } \\
\hline & $\begin{array}{c}T_{\mathrm{g}} \\
{\left[{ }^{\circ} \mathbf{C}\right]}\end{array}$ & $\begin{array}{c}E_{G}^{\prime} \\
{[\mathrm{MPa}]}\end{array}$ & $\begin{array}{c}E_{\mathrm{R}}^{\prime} \\
{[\mathrm{MPa}]}\end{array}$ & $\begin{array}{c}M_{\mathrm{c}} \\
{[\mathrm{g} / \mathrm{mol}]}\end{array}$ & $\begin{array}{c}T_{\mathrm{g}} \\
{\left[{ }^{\circ} \mathbf{C}\right]}\end{array}$ & $\begin{array}{c}E_{G}^{\prime} \\
{[\mathrm{MPa}]}\end{array}$ & $\begin{array}{c}E_{\mathrm{R}}^{\prime} \\
{[\mathrm{MPa}]}\end{array}$ & $\begin{array}{c}M_{\mathrm{c}} \\
{[\mathrm{g} / \mathrm{mol}]}\end{array}$ & $\begin{array}{c}T_{\mathrm{g}} \\
{\left[{ }^{\circ} \mathbf{C}\right]}\end{array}$ & $\begin{array}{c}E_{G}^{\prime} \\
{[\mathrm{MPa}]}\end{array}$ & $\begin{array}{c}E_{\mathrm{R}}^{\prime} \\
{[\mathrm{MPa}]}\end{array}$ & $\begin{array}{c}M_{\mathbf{c}} \\
{[\mathrm{g} / \mathrm{mol}]}\end{array}$ \\
\hline $\mathrm{U}$ & 113 & 2480 & 29.5 & 669 & 92 & 2503 & 8.3 & 1057 & 119 & 2976 & 18.8 & 607 \\
\hline W23 & 108 & 2525 & 36.1 & 736 & 84,91 & 2752 & 6.4 & 1207 & 110,115 & 3078 & 12.7 & 682 \\
\hline W50 & 108,133 & 2265 & 30.7 & 597 & 81,95 & 2405 & 8.6 & 1182 & 103,122 & 2520 & 18.7 & 678 \\
\hline W70 & 112,132 & 2084 & 27.2 & 582 & 83,94 & 2753 & 6.0 & 1161 & 98,125 & 2927 & 14.8 & 691 \\
\hline UVI & 125 & 2625 & 29.8 & 557 & 94 & 3073 & 15.7 & 995 & 120 & 3340 & 21.9 & 598 \\
\hline UVII & 126 & 2843 & 32.0 & 549 & 95 & 2637 & 10.1 & 977 & 125 & 2973 & 23.7 & 560 \\
\hline
\end{tabular}

distribution in the length of the polymer chains and thus inhomogeneity in the obtained network and higher free volume [46]. As illustrated in Figure 4b, and Table 2, the tan $\delta$ peak broadens after exposure at water of $23^{\circ} \mathrm{C}$, and shifts towards lower temperatures (by $5^{\circ} \mathrm{C}$ ). On the contrary, exposure in higher water temperatures results in two peaks, the former at lower position compared to the original $T_{\mathrm{g}}$ (up to $5^{\circ} \mathrm{C}$ shift), and the latter at significantly higher temperature (up to $20^{\circ} \mathrm{C}$ shift). These results can be well explained considering the dual role of the water molecule in epoxy systems. As extensively discussed ingress of higher mobility water may result in plasticization of the resin and/or chain scission/leaching of partially cured molecules and in turn lowering of the $T_{\mathrm{g}}[24-26,45]$. Increase of the $T_{\mathrm{g}}$ has been also documented after water immersion and may be connected with either continuation of the crosslinking in partially cured systems and/or formation of a secondary network between water molecules and polar groups of the epoxy system [24-26, 45]. Thus the broadening and even split of the $\tan \delta$ peak around the $T_{\mathrm{g}}$ upon exposure implies that both aforementioned mechanisms take place during exposure of the SC system.

Based on the response presented in Figure $4 \mathrm{c}$ and the viscoelastic data in Table 2, it can be deduced that the unexposed MC system possesses similar $E_{G}^{\prime}$ values as those found in the SC one. Furthermore it can be observed that $E^{\prime}{ }_{\mathrm{G}}$ is slightly improved (up to $10 \%$ ) after water exposure. At the same time lower $E_{\mathrm{R}}^{\prime}$ values are found in the MC system after immersion, with the only exception being the system immersed at $50^{\circ} \mathrm{C}$ which presents a slight improvement. As seen in Figure 4d, the unexposed MC system presents a better-defined glass transition peak, which is however located at considerably lower temperature compared to the respective $\mathrm{SC}$ system $\left(92\right.$ vs. $\left.113^{\circ} \mathrm{C}\right)$. These findings suggest that the $\mathrm{MC}$ system is more homogenous, and at the same time less crosslinked compared to the SC system. As in the case of the SC system, water exposure results in broadening/split of the $T_{\mathrm{g}}$ related peak (Figure 4d, Table 2), however the lowering of the $T_{\mathrm{g}}$ is more pronounced (up to $11^{\circ} \mathrm{C}$ after water exposure at $50^{\circ} \mathrm{C}$ ) compared to the limited drop of up to $5^{\circ} \mathrm{C}$ in the case of the SC system. These results imply plasticization, chain scission and/or leaching of partially cured molecules, mechanisms that need to be confirmed based on the crosslinking properties of the system [24-26, 45].

Finally, the unexposed FC system presents $E_{G}^{\prime}$ values close to $3000 \mathrm{MPa}$, and a relatively broad glassrubbery transition at $119^{\circ} \mathrm{C}$ (see Figures $4 \mathrm{e}$ and $4 \mathrm{f}$ ). Next to that, a shoulder is observed in the $\tan \delta$ curve of the system at app. $90^{\circ} \mathrm{C}$. Compared to the $\mathrm{SC}$ and $\mathrm{MC}$ systems, the higher $E_{\mathrm{G}}^{\prime}$ and $T_{\mathrm{g}}$ values obtained here imply that the FC system has domains with high crosslink density. At the same time, the existence of the $\tan \delta$ shoulder at lower temperature suggests that this system is inhomogeneous, and contains also a considerable amount of partially cured molecules. This finding can be connected with the limited time of curing of the FC system. Upon immersion the FC system shows an up to $15 \%$ reduction of the $E^{\prime}{ }_{\mathrm{G}}$ and $32 \%$ of $E_{\mathrm{R}}^{\prime}$ values, which however do not vary monotonically with the water temperature (Figure 4e, Table 2). Similarly to the MC system, two $T_{\mathrm{g}}$ related peaks can be found after water exposure, the one being up to $21^{\circ} \mathrm{C}$ lower to the $T_{\mathrm{g}}$ of the unexposed system and the other up to $6^{\circ} \mathrm{C}$ higher after immersion in water of higher temperatures (Figure 4f). Furthermore, it is interesting to note that the shoulder 
found at $90^{\circ} \mathrm{C}$ before exposure, is shifted to $70^{\circ} \mathrm{C}$ after water exposure at $23^{\circ} \mathrm{C}$. As in the case of the $\mathrm{SC}$ system we see also here the existence of the two competitive mechanisms of water, the one that leads to plasticization, chain scission and/or leaching of partially cured molecules, and the other that supports the formation of a secondary network and/or the continuation of the crosslinking process [24-26, 45]. It seems, however, that due to partial curing of the FC system the former prevails over the latter.

Further to the previous analysis, an attempt is made here to define the crosslinking properties of the investigated systems based on the DMA data. Several approaches have been proposed in the past to calculate the average crosslink density $(v)$ and the mean molecular weight between the crosslinks $\left(M_{\mathrm{c}}\right)$ of polymers [8, 44-49]. Using the theory of rubber elasticity this calculation is possible based on the rubbery plateau modulus $\left(E_{\mathrm{R}}^{\prime}\right)$ well above the $T_{\mathrm{g}}$. This methodology has been employed to calculate the $M_{\mathrm{c}}$ and $v$ of toughened epoxies [46-48]; however it should be noted that limitations arise from the use of the kinetic theory of rubber in very highly crosslinked system. Another way to calculate the $M_{\mathrm{c}}$, is via the shift in the $T_{\mathrm{g}}$ according to Equation (4) [44]:

$$
M_{\mathrm{c}}=\frac{39}{T_{\mathrm{g}}-T_{\mathrm{g} 0}}
$$

where $M_{\mathrm{c}}$ is the mean molecular weight between the crosslinks in $[\mathrm{kg} / \mathrm{mol}]$, and $T_{\mathrm{g} 0}$ is the glass transition temperature of the linear polymer taken as $55^{\circ} \mathrm{C}[8$, 49]. This semi-empirical equation has been validated for both slow and fast-curing resins $[8,49]$ and has been employed here for the calculation of $M_{\mathrm{c}}$. As aforementioned, water exposure results in broadening and even split of the $\tan \delta$ peak that corresponds to the $T_{\mathrm{g}}$. For the estimation of $M_{\mathrm{c}}$, a mean value of $T_{\mathrm{g}}$ is considered here. It should be noted that since $T_{\mathrm{g} 0}$ is taken from other studies, the obtained $M_{\mathrm{c}}$ values are only valid for the comparison of each individual system before and after exposure.

As seen in Table 2, $M_{\mathrm{c}}$ of the SC system increases after immersion at $23^{\circ} \mathrm{C}$, and then it monotonically drops with immersion in water of higher temperature (up to $13 \%$ ). Since $M_{\mathrm{c}}$ is inversely proportional to the crosslink density $(v)$, the previous observation implies plasticization, chain scission and/or leaching of partially cured molecules after immersion at $23{ }^{\circ} \mathrm{C}$ and either continuation of crosslinking or creation of hydrogen bonds between water and epoxy at higher temperatures. As FTIR data are not conclusive regarding potential intermolecular hydrogen bonds upon exposure, we suggest that the increase in the crosslink density is associated with continuation of the crosslinking process. The MC and FC systems on the other hand show higher $M_{\mathrm{c}}$ values after water immersion at all temperatures compared to the unexposed specimens (up to $14 \%$ ). In the MC system $M_{\mathrm{c}}$ drops after immersion at higher temperatures, while in FC it fluctuates with water temperature. The higher $M_{\mathrm{c}}$ after exposure in the MC and FC systems is due to plasticization, chain scission, and/or leaching of partially cured molecules, as evidenced by the experimental $\tan \delta$ data. As the immersion temperature increases, further crosslinking takes place, which is however not extensive due to the high temperature curing nature of the MC and FC systems. Overall there is a competition between crosslinking and plasticization/scission during immersion, which is in favor of the former in the SC system, and of the latter in the $\mathrm{MC}$ and, more importantly in the FC systems.

As aforementioned, various studies connect the water absorption with the $T_{\mathrm{g}}$ or the crosslink density $/ M_{\mathrm{c}}$ as well as the secondary interactions between water molecules and polar groups of the resin systems. However, looking at the obtained results, no correlation can be found here since the MC and FC systems contain approximately the same amount of water after exposure, while their $M_{\mathrm{c}}, T_{\mathrm{g}}$ values differ greatly. At the same time, FTIR data do not provide evidence of interactions of water with the polar groups of any of the investigated systems. The only logical explanation behind the obtained behavior is related to the distribution of the chains before exposure, which seems to be very high in the SC system compared to the MC one. This inhomogeneity is expected to create higher free volume, which may explain the increased susceptibility of this system to water. Similarly the FC system was also expected to have high free volume, based on its $M_{\mathrm{c}} / T_{\mathrm{g}}$ values and thus higher susceptibility to water compared to the MC one. We suggest that the discrepancy found here is related to the two network domains of the FC system, i.e. the one that is highly crosslinked and contributes towards higher free volume, and thus higher water absorption and the other that is partially crosslinked and results locally to lower free volume. This, along with the chain scission and/or leaching of the partially cured molecules leads to overall similar water absorption values for the FC and MC systems. 


\subsection{Combined moisture, temperature, and UV exposure}

The effect of combined exposure (moisture - temperature - UV) on the molecular conformations of $\mathrm{SC}, \mathrm{MC}$ and FC systems, as a function of exposure duration, is shown in Figures 5, respectively. Unlike the case of hygrothermal exposure, combined UV radiation/temperature and moisture results in severe alteration of the spectra for all three investigated systems, while similarities can be found in the pattern of SC, MC, and FC systems after exposure. The first key observation is related to the bands at 1036 and $1110 \mathrm{~cm}^{-1}$ of the unexposed systems that unify to a new band at $1060 \mathrm{~cm}^{-1}$ after combined exposure. These bands are related to the reactivity of the ether group, and the observed transformation is linked to photooxidation [50, 51]. The next main alteration of the spectra can be found in the band around $1245 \mathrm{~cm}^{-1}$ which is related to the oxirane ring stretching of the epoxy [50-53]. It can be noted that this band appears only as a shoulder, and its intensity diminishes after combined exposure due to the UV absorbing capacity of the amine cured epoxy resins [53]. Another interesting peak is the one found at app. $1450 \mathrm{~cm}^{-1}$ which is connected with the vibration of the $\mathrm{C}=\mathrm{C}$ bond of the $-\mathrm{CH}_{2}$ unit $[35,36]$. As observed after the combined UV exposure this band diminishes in all three systems, suggesting that most of the $\mathrm{C}=\mathrm{C}$ groups have been crosslinked as discussed before [36]. Furthermore the combined UV exposure does not bring new peaks at 1660 and $1730 \mathrm{~cm}^{-1}$ in the investigated spectra, which are indicative of formation of amide and ketone due extensive degradation $[51,53]$.

In order to evaluate the effect of the combined exposure (moisture - temperature - UV) on the mechanical performance of the three investigated systems, representative stress-strain curves of selected specimens before and after exposure are presented in Figure 6. As illustrated in Figure 6a, the SC system presents an elastic region followed by some plastic deformation before exposure. As expected, the combined UV exposure results in embrittlement of the SC resin, illustrated by a significant reduction of the strain at break and a slight increase in the $E$ modulus. The MC system is slightly more ductile before exposure and becomes more brittle after UV exposure (Figure 6b). Finally, the FC system is less ductile before exposure and presents a great reduction in strength and strain at break after exposure (Figure 6c). Furthermore, it
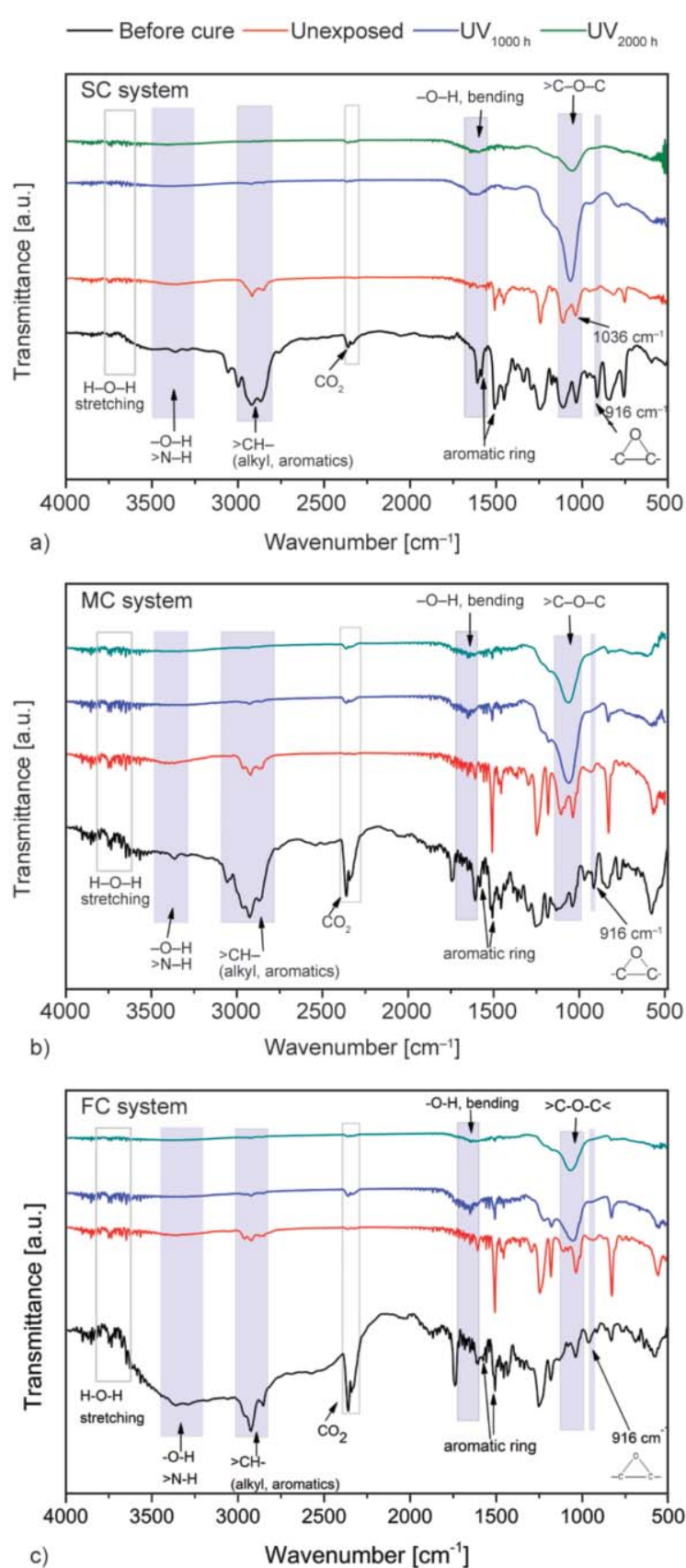

Figure 5. FTIR-ATR spectra of a) SC, b) MC and c) FC systems before and after UV exposure; spectra of uncured systems are added for reference.

seems that deterioration is almost completed after $1000 \mathrm{~h}$ of combined exposure, and further exposure has little influence on the residual properties of the FC system.

For a direct comparison of the effect of combined UV exposure on the response of the $\mathrm{SC}, \mathrm{MC}$ and $\mathrm{FC}$ systems, average values of $E$-modulus, tensile strength, and strain at break [\%] are calculated and presented in Figure 7. As seen in Figure 7, the FC system has the highest $E$-modulus and strength and the lowest strain at break before exposure while the SC system 

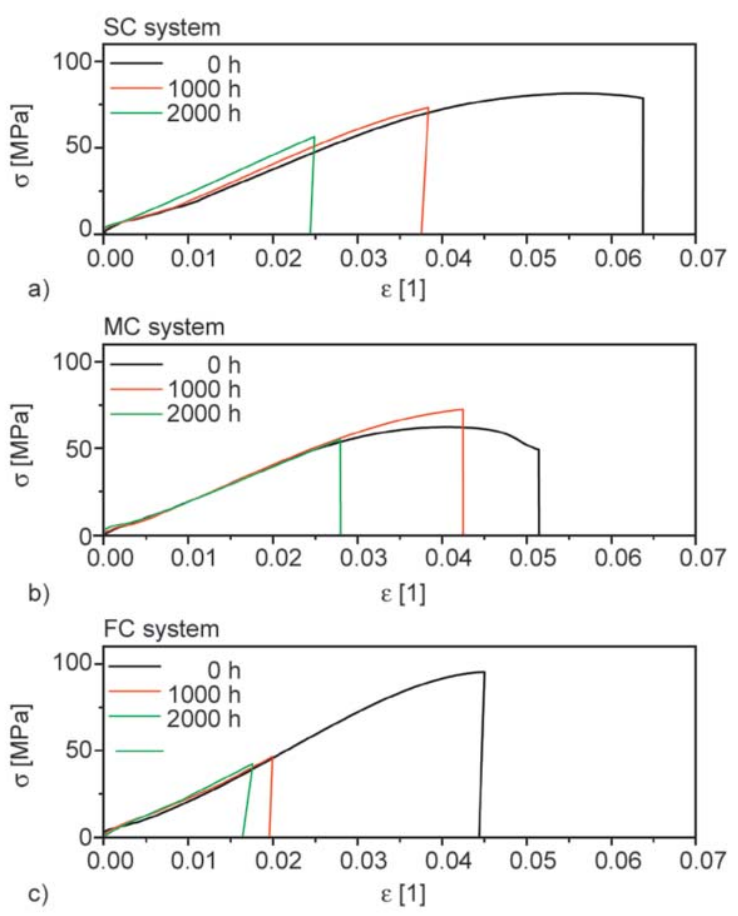

Figure 6. Representative stress - strain curves of a) SC, b) $\mathrm{MC}$ and c) FC systems before and after UV exposure.

shows the same $E$-modulus and considerably higher strain at break compared to the MC system. E-Modulus steadily increases with exposure duration for the SC system (up to $18 \%$ ), it remains almost constant for the MC one (small variations lay within the experimental error) while it slightly drops in the case of the $\mathrm{FC}$ resin (up to 5\%). UV exposure leads to $12 \%$ decline of strength in the SC system and $20 \%$ in MC one. (Figure 7b). UV is however detrimental for the strength of the FC resin since it leads in $40 \%$ loss of the strength after $1000 \mathrm{~h}$ and $50 \%$ after $2000 \mathrm{~h}$ of exposure. Strain at break is also considerably deteriorated in the $\mathrm{SC}, \mathrm{MC}$, and $\mathrm{FC}$ systems, which show up to 40,50 , and $56 \%$ reduction after $2000 \mathrm{~h}$ of exposure, respectively. During combined UV exposure antagonistic effects (i.e. continuation of the crosslinking process, chain scission etc.) take place that influences the mechanical performance of the investigated systems. The small increase of the $E$-modulus of the $\mathrm{SC}$ and the strength of the MC system implies a continuation of crosslinking process during exposure, while the opposite trend followed by the FC system can be linked with chain scission processes.

As aforementioned DMA can provide useful information around the viscoelastic response and especially the $T_{\mathrm{g}}$ and the crosslink density of the investigated systems before and after combined UV exposure. Thus, Figure 8 illustrates the variation of $E^{\prime}$ and
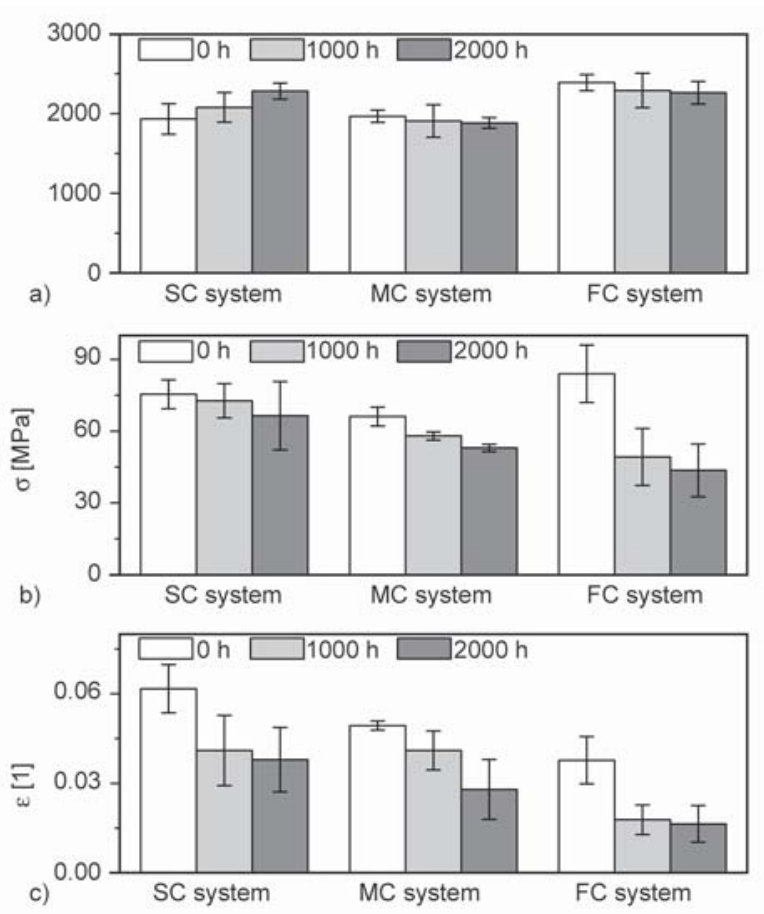

Figure 7. a) Young's modulus $(E)$, b) tensile strength $(\sigma)$ and c) strain at break $(\varepsilon)$ of $\mathrm{SC}, \mathrm{MC}$ and $\mathrm{FC}$ systems before and after UV exposure.

$\tan \delta$ as a function of temperature for SC (Figures 8a, 8b), MC (Figures 8c, 8d) and FC (Figures 8e, 8f), systems, respectively, while Table 2 depicts the $E_{\mathrm{G}}^{\prime}$, $E_{\mathrm{R}}^{\prime}, T_{\mathrm{g}}$ and the calculated $M_{\mathrm{c}}$ values before and after combined UV exposure.

Upon combined UV exposure, a clear increase in the $T_{\mathrm{g}}$ of the SC system is obtained, which is illustrated by a shift of $E^{\prime}$ and $\tan \delta$ curves in the glass to rubbery transition zone by $12-13{ }^{\circ} \mathrm{C}$ (see Figures $8 \mathrm{a}, 8 \mathrm{~b}$ and Table 2). At the same time $E^{\prime}{ }_{\mathrm{G}}$ and $E^{\prime}{ }_{\mathrm{R}}$ values present an up to $15-18 \%$ increase, which is more pronounced after $2000 \mathrm{~h}$ of UV radiation. The MC system also presents an increase of the $E^{\prime}$ in both glassy (up to $12 \%$ ) and rubbery states (up to $26 \%$ ) and a $2-3{ }^{\circ} \mathrm{C}$ increase of the $T_{\mathrm{g}}$ upon exposure. Interestingly the $E^{\prime}$ and $\tan \delta$ curves of the UV radiated specimens do not follow the same variation with the one found in the unexposed ones. As observed, just above the $T_{\mathrm{g}}$ the $E^{\prime}$ curve presents a small shoulder at 130 $135^{\circ} \mathrm{C}$, which is more pronounced after $2000 \mathrm{~h}$ of UV exposure. Similarly the tan $\delta$ curve does not show the gradual drop that is usually found in this region. Finally, the FC system is also improved in both glassy and rubbery region in terms of $E^{\prime}$. This system does not show any increase of the $T_{\mathrm{g}}$ after $1000 \mathrm{~h}$ of $\mathrm{UV}$ exposure; however it can be clearly observed that the shoulder in the tan $\delta$ curve of the unexposed specimen (at $90^{\circ} \mathrm{C}$ ), is eliminated and the $T_{\mathrm{g}}$ peak is symmetric 


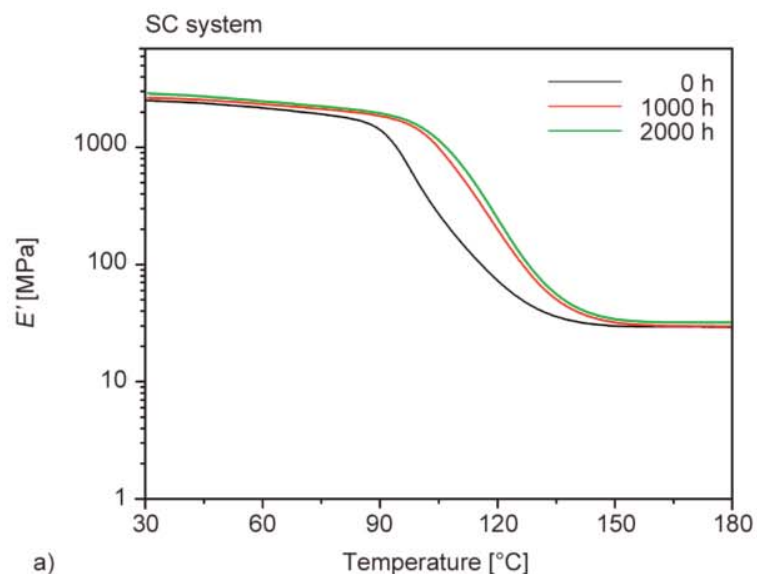

a)

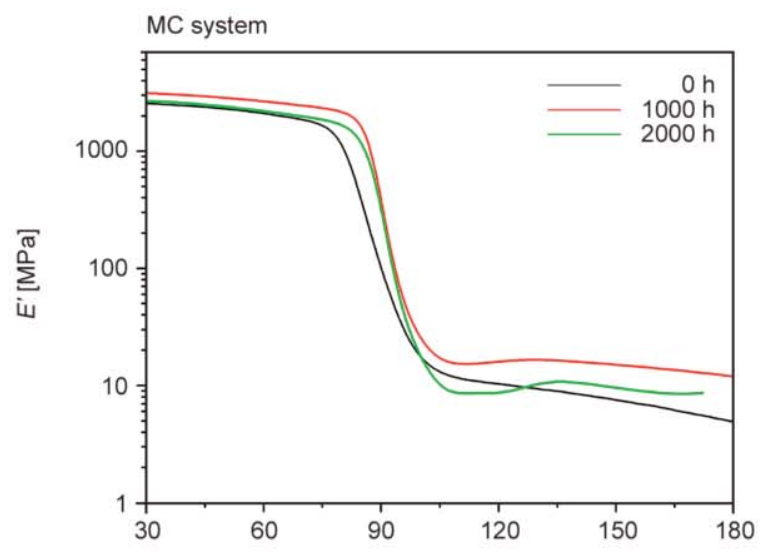

c)

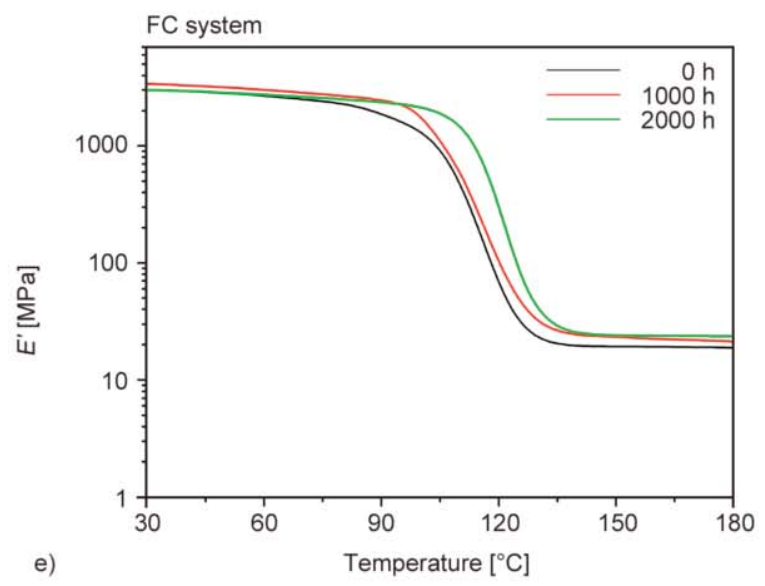

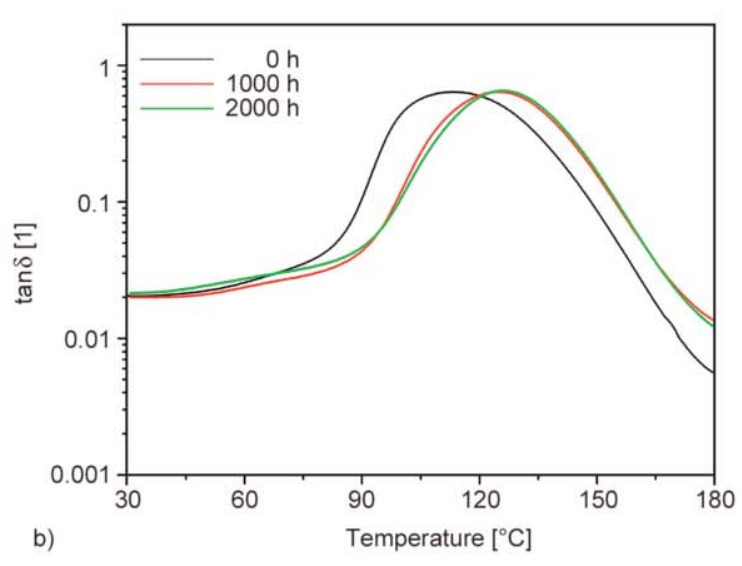

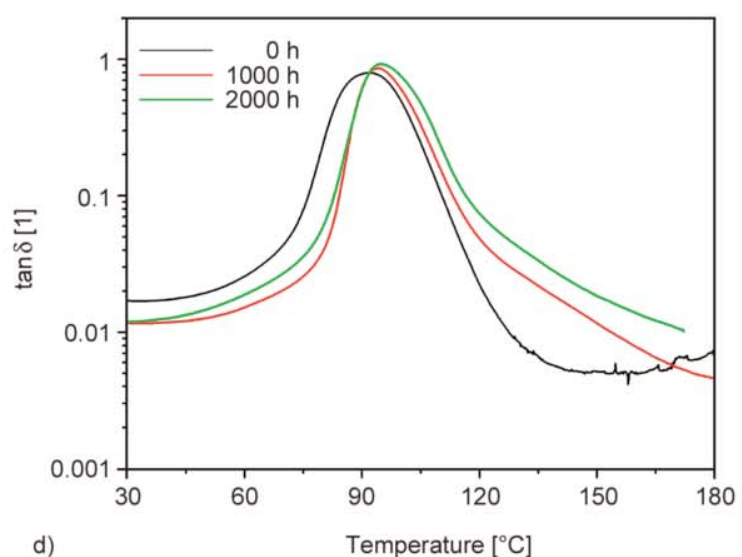

d)

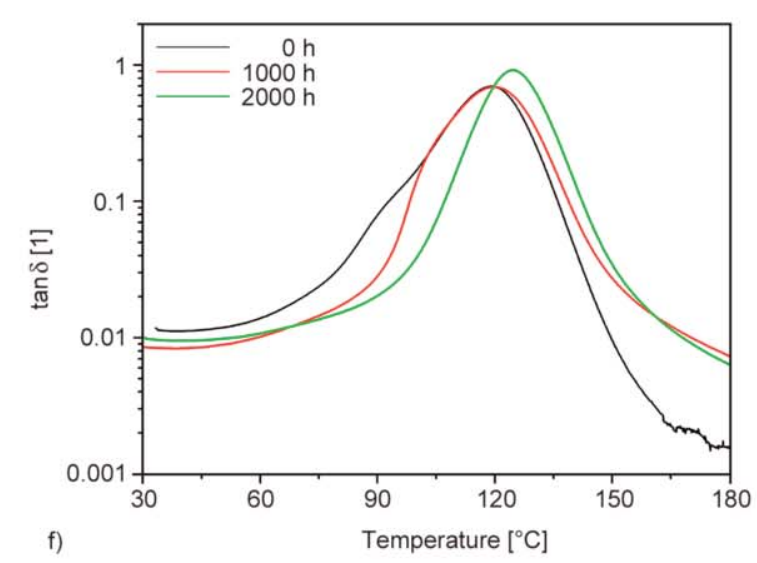

Figure 8. Storage modulus $\left(E^{\prime}\right)$ and loss factor $(\tan \delta)$ of SC: a), b), MC: c), d) and FC: e), f) systems, respectively before and after UV exposure of different durations.

after radiation. Further UV exposure results in the shift of the $T_{\mathrm{g}}$ related peak to higher temperatures. Based on the result of Table 2, it is deduced that UV exposure results in lower $M_{\mathrm{c}}$ values by up to $18 \%$ in the case of the SC system and by up to $8 \%$ for MC and $\mathrm{FC}$ systems. It should be however noted that the calculation of $M_{\mathrm{c}}$ does not account any secondary peaks.

The viscoelastic response of the investigated systems after UV exposure supports the findings of the water exposure analysis. Firstly, the elimination of the shoulder in the case of the FC system, and the variation of the $\tan \delta$ curve above the $T_{\mathrm{g}}$ of the MC system clearly suggests the existence of partially cured monomers and the continuation of the crosslinking during the UV exposure. At the same time, the SC system exhibits the greatest drop in $M_{\mathrm{c}}$ (i.e. increase in crosslink density) after exposure which indicates that this system has the greatest potential for hardening upon exposure. This might explain why the SC 
system maintains better its mechanical properties upon UV radiation.

\section{Conclusions}

The current study investigates the degradation behavior of conventional and fast curing epoxy resin systems after hygrothermal exposure and combined UV radiation by means of water absorption, FTIR, mechanical and thermomechanical characterization. It has been shown that curing is crucial for the environmental response of the investigated systems. More specifically it has been shown that:

- The SC system absorbs up to 1.5 higher amounts of water compared to the MC and FC ones. This has been linked to the wide distribution of polymer chains found in the SC system that creates excess free volume.

- MC and FC systems present similar water absorption values, although they considerably differ in terms of viscoelastic properties. This has been associated with the two network domains of the FC system.

- Compared to the MC and FC systems, the SC system has the greatest potential for hardening upon exposure. This is due to the fact that environmental exposure conditions better match the curing requirements of this system.

- Instead of curing, water exposure, especially at lower temperatures, results in plasticization, chain scission, and even leaching of any partially cured molecules in the MC and FC resins since continuation of crosslinking is not favored by the environmental conditions.

- The SC system maintains better its mechanical properties upon UV exposure since continuation of crosslinking prevails over chain scission.

Although FTIR has been used in the past to follow the degradation of epoxy resins, DMA seems more powerful in the sense that it identifies changes in the chain conformation/mobility of the polymer network upon exposure.

\section{Acknowledgements}

We would like to acknowledge the contribution of L. Mavrodimos, A. Alexandridis and D. Tsiklitari in the execution of the experimental program of the current study within the framework of their final year thesis at the Department of Materials Science and Eng., University of Ioannina, Greece and Huntsman Advanced Materials $\mathrm{GmbH}$, Basel for kindly providing the tested resin systems.

\section{References}

[1] Jin F-L., Li X., Park S-J.: Synthesis and application of epoxy resins: A review. Journal of Industrial and Engineering Chemistry, 29, 1-11 (2015).

https://doi.org/10.1016/j.jiec.2015.03.026

[2] Duemichen E., Javdanitehran M., Erdmann M., Trappe V., Sturm H., Braun U., Ziegmann G.: Analyzing the network formation and curing kinetics of epoxy resins by in situ near-infrared measurements with variable heating rates. Thermochimica Acta, 616, 49-60 (2015). https://doi.org/10.1016/j.tca.2015.08.008

[3] Lakho D. A., Yao D., Cho K., Ishaq M., Wang Y.: Study of the curing kinetics toward development of fast-curing epoxy resins. Polymer - Plastics Technology and Engineering, 56, 161-170 (2017). https://doi.org/10.1080/03602559.2016.1185623

[4] Zhao X., Huang Z., Song P., Yang H., Zhang Y.: Curing kinetics and mechanical properties of fast curing epoxy resins with isophorone diamine and $\mathrm{N}$-(3-aminopropyl)imidazole. Journal of Applied Polymer Science, 136, 47950/1-47950/10 (2019).

https://doi.org/10.1002/app.47950

[5] Xu Y-J., Wang J., Tan Y., Qi M., Chen L., Wang Y-Z.: A novel and feasible approach for one-pack flame-retardant epoxy resin with long pot life and fast curing. Chemical Engineering Journal, 337, 30-39 (2018). https://doi.org/10.1016/j.cej.2017.12.086

[6] Yang B., Mao Y., Zhang Y., Wei Y., Liu W., Qiu Y.: Fast-curing halogen-free flame-retardant epoxy resins and their application in glass fiber-reinforced composites. Textile Research Journal, 18, 3700-3707 (2018). https://doi.org/10.1177/0040517518819845

[7] Bernath A., Kärger L., Henning F.: Accurate cure modeling for isothermal processing of fast curing epoxy resins. Polymers, 8, 390/1-390/19 (2016).

https://doi.org/10.3390/polym8110390

[8] Keller A., Masania K., Taylor A. C., Dransfeld C.: Fastcuring epoxy polymers with silica nanoparticles: Properties and rheo-kinetic modelling. Journal of Materials Science, 51, 236-251 (2015). https://doi.org/10.1007/s10853-015-9158-y

[9] Wang Y., Liu W., Qiu Y., Wei Y.: Single-component and fast-curing epoxy resin for liquid composite molding processes. MATEC Web of Conferences, 130, 07005/107005/5 (2017).

https://doi.org/10.1051/matecconf/201713007005

[10] Hayaty M., Honarkar H., Beheshty M. H.: Curing behavior of dicyandiamide/epoxy resin system using different accelerators. Iranian Polymer Journal, 22, 591598 (2013). https://doi.org/10.1007/s13726-013-0158-y

[11] Sprenger S., Kothmann M. H., Altstaedt V.: Carbon fiber-reinforced composites using an epoxy resin matrix modified with reactive liquid rubber and silica nanoparticles. Composites Science and Technology, 105, 8695 (2014).

https://doi.org/10.1016/j.compscitech.2014.10.003 
[12] Hinkley J. A., Connell J. W.: Resin systems and chemistry: Degradation mechanisms and durability. in 'Longterm durability of polymeric matrix composites' (eds.: Pochiraju K., Tandon G., Schoeppner G.) Springer, Boston, 1-37 (2012).

https://doi.org/10.1007/978-1-4419-9308-3 1

[13] Apicella A., Nicolais L., Nobile M. R., CastiglioneMorelli M. A.: Effect of processing variables on the durability of epoxy resins for composite systems. Composites Science and Technology, 24, 101-121 (1985). https://doi.org/10.1016/0266-3538(85)90054-5

[14] Awad S. A., Fellows C. M., Mahini S. S.: Effects of accelerated weathering on the chemical, mechanical, thermal and morphological properties of an epoxy/multiwalled carbon nanotube composite. Polymer Testing, 66, 70-77 (2018).

https://doi.org/10.1016/j.polymertesting.2017.12.015

[15] Frigione M., Lettieri M.: Durability issues and challenges for material advancements in FRP employed in the construction industry. Polymers, 10, 247/1-247/15 (2018). https://doi.org/10.3390/polym10030247

[16] Helbling C., Karbhari V. M.: Durability of composites in aqueous environments. in 'Durability of composites for civil structural applications' (eds.: Karbhari V. M.) Woodhead, Cambridge, 31-71 (2007). https://doi.org/10.1533/9781845693565.1.31

[17] Xiao G. Z., Shanahan M. E. R.: Water absorption and desorption in an epoxy resin with degradation. Journal of Polymer Science Part B: Polymer Physics, 35, 26592670 (1997).

https://doi.org/10.1002/(SICI)10990488(19971130)35:16<2659::AID-POLB9>3.0.CO;2-K

[18] Dao B., Hodgkin J. H., Krstina J., Mardel J., Tian W.: Accelerated ageing versus realistic ageing in aerospace composite materials. IV. Hot/wet ageing effects in a low temperature cure epoxy composite. Journal of Applied Polymer Science, 106, 4264-4276 (2007).

https://doi.org/10.1002/app.27104

[19] Tcherbi-Narteh A., Hosur M., Triggs E., Jeelani S.: Thermal stability and degradation of diglycidyl ether of bisphenol A epoxy modified with different nanoclays exposed to UV radiation. Polymer Degradation and Stability, 98, 759-770 (2013).

https://doi.org/10.1016/j.polymdegradstab.2012.12.013

[20] Tcherbi-Narteh A., Hosur M., Triggs E., Owuor P., Jelaani S.: Viscoelastic and thermal properties of full and partially cured DGEBA epoxy resin composites modified with montmorillonite nanoclay exposed to UV radiation. Polymer Degradation and Stability, 101, 81-91 (2014).

https://doi.org/10.1016/j.polymdegradstab.2013.12.033

[21] ASTM D570-98(2018): Standard test method for water absorption of plastics (2018).

https://doi.org/10.1520/D0570-98R18
[22] Johncock P., Tudgey G. F.: Some effects of structure, composition and cure on the water absorption and glass transition temperature of amine-cured epoxies. British Polymer Journal, 18, 292-302 (1986).

https://doi.org/10.1002/pi.4980180504

[23] Chaplin A., Hamerton I., Herman H., Mudhar A. K., Shaw S. J.: Studying water uptake effects in resins based on cyanate ester/bismaleimide blends. Polymer, 41, 3945-3956 (2000). https://doi.org/10.1016/S0032-3861(99)00603-5

[24] Gkikas G., Douka D-D., Barkoula N-M., Paipetis A. S.: Nano-enhanced composite materials under thermal shock and environmental degradation: A durability study. Composites Part B: Engineering, 70, 206-214 (2015). https://doi.org/10.1016/j.compositesb.2014.11.008

[25] Starkova O., Buschhorn S. T., Mannov E., Schulte K., Aniskevich A.: Water transport in epoxy/MWCNT composites. European Polymer Journal, 49, 2138-2148 (2013).

https://doi.org/10.1016/j.eurpolymj.2013.05.010

[26] Zhou J., Lucas J. P.: Hygrothermal effects of epoxy resin. Part I: The nature of water in epoxy. Polymer, 40, 5505-5512 (1999).

https://doi.org/10.1016/S0032-3861(98)00790-3

[27] ASTM D4329-13: Standard practice for fluorescent ultraviolet (UV) lamp apparatus exposure of plastics (2013). https://doi.org/10.1520/D4329-13

[28] Crank J.: The mathematics of diffusion. Oxford University Press, London (1975).

[29] Jana S., Zhong W-H.: FTIR study of ageing epoxy resin reinforced by reactive graphitic nanofibers. Journal of Applied Polymer Science, 106, 3555-3563 (2007). https://doi.org/10.1002/app.26925

[30] Smith R. E., Larsen F. N., Long C. L.: Epoxy resin cure. II. FTIR analysis. Journal of Applied Polymer Science, 29, 3713-3726 (1984). https://doi.org/10.1002/app.1984.070291207

[31] Wang L. W., Fernando G. F.: Cure monitoring of epoxy resin by simultaneous DSC/FTIR. Advanced Materials Research, 881-883, 905-908 (2014).

https://doi.org/10.4028/www.scientific.net/AMR.881-883.905

[32] Pandita S. D., Wang L., Mahendran R. S., MacHavaram V. R., Irfan M. S., Harris D., Fernando G. F.: Simultaneous DSC-FTIR spectroscopy: Comparison of crosslinking kinetics of an epoxy/amine resin system. Thermochimica Acta, 543, 9-17 (2012).

https://doi.org/10.1016/j.tca.2012.04.024

[33] Cañavate J., Colom X., Pagès P., Carrasco F.: Study of the curing process of an epoxy resin by FTIR spectroscopy. Polymer-Plastics Technology and Engineering, 39, 937-943 (2000). https://doi.org/10.1081/PPT-100101414 
[34] González M. G., Cabanelas J. C., Baselga J.: Applications of FTIR on epoxy resins -Identification, monitoring the curing process, phase separation and water uptake. in 'Infrared spectroscopy - Materials science, engineering and technology' (ed.: Theophanides T.) IntechOpen, London, 261-284 (2012).

https://doi.org/10.5772/36323

[35] Jiang X., He S., Li S., Bai Y., Shao L.: Penetrating chains mimicking plant root branching to build mechanically robust, ultra-stable $\mathrm{CO}_{2}$-philic membranes for superior carbon capture. Journal of Materials Chemistry A, 7, 16704-16711 (2019).

https://doi.org/10.1039/C9TA03416A

[36] Jiang X., Li S., He S., Bai Y., Shao L.: Interface manipulation of $\mathrm{CO}_{2}$-philic composite membranes containing designed UiO-66 derivatives towards highly efficient $\mathrm{CO}_{2}$ capture. Journal of Materials Chemistry A, 6, 15064-15073 (2018).

https://doi.org/10.1039/C8TA03872D

[37] Zhang Y., Sun H., Sadam H., Liu Y., Shao L.: Supramolecular chemistry assisted construction of ultra-stable solvent-resistant membranes for angstrom-sized molecular separation. Chemical Engineering Journal, 371, 535-543 (2019).

https://doi.org/10.1016/j.cej.2019.04.096

[38] Xiao G. Z., Delamar M., Shanahan M. E. R.: Irreversible interactions between water and DGEBA/DDA epoxy resin during hygrothermal aging. Journal of Applied Polymer Science, 65, 449-458 (1997).

https://doi.org/10.1002/(SICI)10974628(19970718)65:3<449::AID-APP4>3.0.CO;2-H

[39] Cotugno S., Mensitieri G., Musto P., Sanguigno L.: Molecular interactions in and transport properties of densely cross-linked networks: A time-resolved FT-IR spectroscopy investigation of the epoxy $/ \mathrm{H}_{2} \mathrm{O}$ system. Macromolecules, 38, 801-811 (2005).

https://doi.org/10.1021/ma040008j

[40] Liu M., Wu P., Ding Y., Chen G., Li S.: Two-dimensional (2D) ATR-FTIR spectroscopic study on water diffusion in cured epoxy resins. Macromolecules, 35, 5500-5507 (2002). https://doi.org/10.1021/ma011819f

[41] Wu P., Siesler H. W.: Water diffusion into epoxy resin: A 2D correlation ATR-FTIR investigation. Chemical Physics Letters, 374, 74-78 (2003). https://doi.org/10.1016/S0009-2614(03)00691-2

[42] Kim H., Urban M. W.: Molecular level chain scission mechanisms of epoxy and urethane polymeric films exposed to $\mathrm{UV} / \mathrm{H}_{2} \mathrm{O}$. Multidimensional spectroscopic studies. Langmuir, 16, 5382-5390 (2000).

https://doi.org/10.1021/la990619i

[43] Ornaghi Jr. H. L., Bolner A. S., Fiorio R., Zattera A. J., Amico S. C.: Mechanical and dynamic mechanical analysis of hybrid composites molded by resin transfer molding. Journal of Applied Polymer Science, 118, 887-896 (2010).

https://doi.org/10.1002/app.32388
[44] Nielsen L. E.: Cross-linking-effect on physical properties of polymers. Journal of Macromolecular Science Part C: Polymer Reviews, 3, 69-103 (1969). https://doi.org/10.1080/15583726908545897

[45] Zhou J., Lucas J. P.: Hygrothermal effects of epoxy resin. Part II: Variations of glass transition temperature. Polymer, 40, 5513-5522 (1999).

https://doi.org/10.1016/S0032-3861(98)00791-5

[46] Karger-Kocsis J., Friedrich K.: Microstructure-related fracture toughness and fatigue crack growth behaviour in toughened, anhydride-cured epoxy resins. Composites Science and Technology, 48, 263-272 (1993). https://doi.org/10.1016/0266-3538(93)90143-5

[47] Karger-Kocsis J., Gremmels J.: Use of hygrothermal decomposed polyester-urethane waste for the impact modification of epoxy resins. Journal of Applied Polymer Science, 78, 1139-1151 (2000).

https://doi.org/10.1002/10974628(20001031)78:5<1139::AID-APP240>3.0.CO;2-Q

[48] Barkoula N-M., Gremmels J., Karger-Kocsis J.: Dependence of solid particle erosion on the cross-link density in an epoxy resin modified by hygrothermally decomposed polyurethane. Wear, 247, 100-108 (2001). https://doi.org/10.1016/S0043-1648(00)00529-9

[49] Bellenger V., Verdu J., Morel E.: Effect of structure on glass transition temperature of amine crosslinked epoxies. Journal of Polymer Science Part B: Polymer Physics, 25, 1219-1234 (1987). https://doi.org/10.1002/polb.1987.090250604

[50] Mailhot B., Morlat-Thérias S., Ouahioune M., Gardette J-L.: Study of the degradation of an epoxy/amine resin, 1. Macromolecular Chemistry and Physics, 206, 575584 (2005). https://doi.org/10.1002/macp.200400395

[51] Rezig A., Nguyen T., Martin D., Sung L., Gu X., Jasmin J., Martin J. W.: Relationship between chemical degradation and thickness loss of an amine-cured epoxy coating exposed to different UV environments. Journal of Coatings Technology and Research, 3, 173-184 (2006). https://doi.org/10.1007/BF02774507

[52] Kumar B. G., Singh R. P., Nakamura T.: Degradation of carbon fiber-reinforced epoxy composites by ultraviolet radiation and condensation. Journal of Composite Materials, 36, 2713-2733 (2002). https://doi.org/10.1177/002199802761675511

[53] Petersen E. J., Lam T., Gorham J. M., Scott K. C., Long C. J., Stanley D., Sharma R., Liddle J. A., Pellegrin B., Nguyen T.: Methods to assess the impact of UV irradiation on the surface chemistry and structure of multiwall carbon nanotube epoxy nanocomposites. Carbon, 69, 194-205 (2014).

https://doi.org/10.1016/j.carbon.2013.12.016 